\title{
Can routine outpatient follow-up of patients with bladder cancer be improved? A multicenter prospective observational assessment of blue light flexible cystoscopy and fulguration
}

This article was published in the following Dove Press journal:

Research and Reports in Urology

\begin{abstract}
Reza Zare'
Magnus Grabe 2

Gregers G Hermann ${ }^{3}$

Per-Uno Malmström ${ }^{4}$

'Department of Urology, Vestre Viken HF Bærum Hospital, Oslo, Norway; ${ }^{2}$ Department of Urology, Skåne University Hospital, University of Lund, Malmö, Sweden; ${ }^{3}$ Department of Urology, Herlev and Gentofte Hospital, Copenhagen University, Denmark; ${ }^{4}$ Department of Urology, Institute of Surgical Sciences, Uppsala University, Uppsala, Sweden
\end{abstract}

\section{Video abstract}

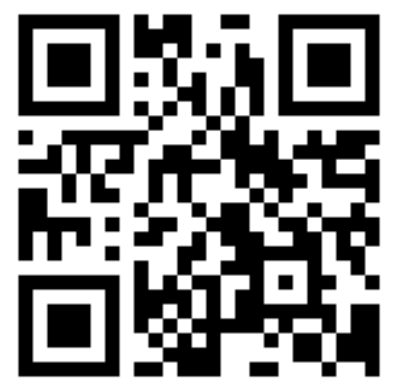

Point your SmartPhone at the code above. If you have a $Q R$ code reader the video abstract will appear. Or use: http://youtu.be/Trp-43RZEBO

Correspondence: Reza Zare Department of Urology, Vestre Viken HF Bærum Hospital, Songneprest Munthekaasveien 1001346 Gjettum, Norway Tel +47267809674

Email reza.zare@vestreviken.no
Background: The aim of this prospective cohort study was to determine the feasibility of incorporating blue light flexible cystoscopy (BLFC) and biopsy/fulguration into routine outpatient follow-up of non-muscle invasive bladder cancer patients.

Methods: The study included patients with non-muscle-invasive bladder cancer (NMIBC) who were scheduled for routine follow-up. Hexaminolevulinate was instilled in the outpatient department, and the bladder was examined under white light and then with BLFC. Biopsies were taken from all suspicious lesions. Small tumors and suspicious lesions were fulgurated on site; patients with larger lesions were referred to the operating room for resection.

Results: The study included 69 patients, with a mean age of 70 years (range 33-89 years) and a mean duration since NMIBC diagnosis of 8 years. Most patients had high-grade cancer at initial diagnosis (52/69) and were at high risk of recurrence (48/69). Two patients per hour could be assessed using outpatient BLFC. Preparation and instillation of hexaminolevulinate took less than 10 minutes per patient, and patients had an additional waiting time of 45-60 minutes following instillation, while the hexaminolevulinate solution was retained in the bladder before examination. Eleven patients had histologically confirmed tumors that were identified using both white light flexible cystoscopy and BLFC. An additional three patients had tumors that were identified by BLFC only: two with Ta tumors and one with carcinoma in situ. Of the 14 patients with confirmed tumors, 11 could be managed on site with fulguration, whereas three were referred to the operating room. No adverse events attributable to BLFC were reported.

Conclusion: Routine outpatient management of patients with NMIBC using BLFC and onsite biopsy/fulguration is feasible, despite the additional time required for hexaminolevulinate instillation, and appears to allow early detection of recurrent lesions, which can be fulgurated without the need for hospitalization.

Keywords: bladder cancer, blue light, diagnosis, flexible cystoscopy, hexaminolevulinate, outpatients

\section{Introduction}

As a result of a high risk of recurrence, ${ }^{1,2}$ patients with non-muscle-invasive bladder cancer (NMIBC) require long-term follow-up, including regular cystoscopy starting at 3 months after tumor resection. ${ }^{1}$ Patients are usually followed up as outpatients, potentially with fulguration or laser removal of small lesions, ${ }^{3}$ but they may require referral to the operating room for resection of larger recurrences. This procedure is 
performed under general anesthesia and requires patients to be hospitalized, which has resource and cost implications. ${ }^{4}$ As a result, bladder cancer is one of the most expensive of all tumors to manage over the long-term. ${ }^{5}$ Hospitalization and repeated follow-up also place a heavy burden on patients, many of whom report impaired quality of life. ${ }^{6,7}$ The ability to identify recurrences while they are still small and can be managed in the outpatient department could therefore substantially reduce the burden of NMIBC follow-up.

Blue light cystoscopy as an adjunct to white light cystoscopy gives higher sensitivity for identifying bladder cancer, including early small lesions, than white light cystoscopy alone. ${ }^{1,8}$ With this approach, a photosensitizing compound (hexaminolevulinate; Hexvix; Photocure ASA, Oslo, Norway) is instilled into the bladder before cystoscopy. This compound is a heme precursor, which preferentially accumulates in tumor cells and fluoresces under blue light, making tumors readily distinguishable from normal mucosa. The sensitivity of hexaminolevulinate-guided blue light cystoscopy with a rigid cystoscope in the operating room has been demonstrated in an extensive program of clinical trials involving more than 1,800 patients. A meta-analysis of patient-level data from clinical trials of hexaminolevulinate showed a significant improvement in the detection of Ta tumors and carcinoma in situ (CIS) compared with white light cystoscopy. ${ }^{9}$ The improved detection of tumors remained apparent regardless of whether the tumors were primary or recurrent and whether patients had low, intermediate, or high risk of recurrence. The risk of recurrence is reduced after blue light cystoscopy compared with white light cystoscopy (relative risk 0.761). ${ }^{9}$ Blue light cystoscopy is therefore recommended as an adjunct to white light cystoscopy in the latest European Association of Urology guidelines ${ }^{1}$ and by experts in Europe, ${ }^{10}$ the USA, ${ }^{11}$ and the Nordic region. ${ }^{12}$ The European expert panel noted that hexaminolevulinate-guided blue light cystoscopy allowed the identification of previously missed or recurrent tumors at follow-up, especially in high-risk patients. ${ }^{10}$

There has been interest in use of blue light cystoscopy with a flexible scope for many years. In early reports, the technique appeared to be at least comparable to white light flexible cystoscopy and slightly inferior to rigid blue light cystoscopy in terms of NMIBC detection rates. ${ }^{13-17}$ However, not all of those studies were in outpatient settings, and they did not use the latest equipment. For example, the first two publications, both from $2005,{ }^{13,14}$ reported experiences with the patient under general anesthesia in the operating room and using flexible blue light fiberscopes, which have noticeably poorer visualization than currently available videoscopes. In the operating room, rigid equipment provides better suction to remove debris and urine, avoiding green autofluorescence from the urine. ${ }^{18}$ Thus, the situation was very different from an outpatient setting with conscious patients.

Given an urgent need to control the cost of bladder cancer management in the current resource-constrained environment, this study was performed to investigate whether use of hexaminolevulinate-guided blue light flexible cystoscopy (BLFC) could enhance outpatient management of patients. The aim of the study was to determine the feasibility of incorporating BLFC into routine outpatient practice, in terms of practical and logistical considerations as well as outcomes such as image and biopsy quality, and the ability to identify recurrent lesions early enough so that they could be fulgurated on site without hospitalization or general anesthesia. This study builds on the single-center experience using the latest technology reported previously by Hermann et al., ${ }^{3,4}$

\section{Materials and methods \\ Patients}

Patients could be included if they had a history of bladder cancer and were attending an outpatient clinic for routine cystoscopic examination. Patients were recruited from two centers in Sweden (Uppsala University Hospital, Uppsala, and Skåne University Hospital, Malmö) and one center in Norway (Vestre Viken, Bærum). All procedures in the study were explained to the patients by their physician, and, before the examination, patients provided written informed consent to receive hexaminolevulinate and undergo BLFC. As this study was intended to assess the practical feasibility of changes in service delivery, and all patients were managed according to accepted clinical practice, protocol registration and ethics committee approval were not required, however patient consent was obtained for both the study and the procedure.

\section{BLFC}

Cystoscopy was undertaken by a physician trained in standard flexible cystoscopy, who performed the procedure and any biopsies or fulguration with the support of an outpatient department nurse. All staff taking part in the study at the different centers was trained in BLFC performance by a urologist with extensive experience, Gregers G Hermann, before patients were enrolled, to ensure consistency across all participating centers.

One hour before cystoscopy was scheduled, a nurse instilled $50 \mathrm{~mL}$ of hexaminolevulinate solution into the patient's bladder using a LoFric catheter CH 12 (Astra Tech AB, Mölndal, Sweden). The patient then left the instillation 
room and could wait without supervision, for example, in a waiting area or cafeteria, or could go for a walk before cystoscopy.

For the outpatient flexible cystoscopy procedure, a prototype high-definition video cystoscope with white and blue light sources (Telecam; Karl Storz, Tuttlingen, Germany) was used to examine the bladder. The bladder was washed before and during cystoscopy with saline or sterile water through the suction canal to remove urine, which gives green autofluorescence when illuminated with blue light, impairing visualization of the bladder. ${ }^{18}$ The bladder was first inspected using high-definition white light flexible cystoscopy, and the number and location of tumors and suspicious areas were noted. After white light flexible cystoscopy was completed, the bladder was re-examined using BLFC, and all fluorescing lesions were documented.

\section{Biopsies and fulguration}

Biopsies were taken from suspicious lesions seen under either white light or blue light, with bladder cancer confirmed histologically. All biopsies were obtained through the flexible cystoscope with bendable single-use biopsy forceps (11027401, Karl Storz) with a working length of 1,200 $\mathrm{mm}$ and a diameter of 5 Fr. Small tumors (eg, $<1 \mathrm{~cm}$ ) and suspicious lesions were fulgurated with patient consent using a $4 \mathrm{Fr}$ Bugbee electrode placed through a 5 Fr working port in the flexible cystoscope, using a diathermy generator at 8-20 W.

\section{Data collection and analysis}

Prospectively collected baseline data included patient information, bladder cancer stage and grade at original diagnosis, estimated risk of recurrence, time since diagnosis of bladder cancer, number of previous cystoscopies, number of previous transurethral resections of the bladder (TURBs), and previous use of intravesical therapy. Observations collected during the study included best practice workflow and the logistics of performing BLFC, the overall ease of use of BLFC, the quality of images and biopsies obtained during cystoscopy, the ability to fulgurate lesions detected during cystoscopy, and the investigators' experiences with the BLFC technology. Logistical parameters included the time taken to prepare and instill hexaminolevulinate solution, patient waiting time between instillation and cystoscopy, and the number of patients who could have BLFC per hour in this study setting. The tolerability of BLFC was assessed in terms of adverse events and the level of patient discomfort during hexaminolevulinate instillation and cystoscopy. These factors were combined to provide an overall assessment of the feasibility of BLFC and biopsy or fulguration in routine outpatient follow-up and management of patients with known NMIBC. As this was a pilot study, no formal statistical significance testing was done.

\section{Results}

The study included 69 patients with NMIBC (22 patients at Uppsala, 22 at Malmö, and 25 at Bærum) who had follow-up BLFC between April and September 2013.

Across the patient population, the mean age was 70 years (range 33-89 years), and the mean duration since diagnosis of bladder cancer was 8 years. Most patients had high-grade cancer at their initial diagnosis (52/69) and were at high risk of disease recurrence (48/69). Patients had undergone a mean of 11 previous cystoscopies and a mean of three previous TURBs for NMIBC. The characteristics of the patient populations at each study center are shown in Table 1.

The time required for the preparation and instillation of the hexaminolevulinate solution was around 10 minutes per patient in all three centers (Table 2). Because hexaminolevulinate needs to be retained in the bladder to ensure adequate uptake before cystoscopy, patients participating in this study

Table I Demographics and characteristics of consenting patients enrolled in the study

\begin{tabular}{|c|c|c|c|c|}
\hline $\begin{array}{l}\text { Demographics and } \\
\text { characteristics }\end{array}$ & Uppsala & Malmö & Bærum & Overall \\
\hline $\begin{array}{l}\text { Patients undergoing blue light } \\
\text { flexible cystoscopy, } n\end{array}$ & 22 & 22 & 25 & 69 \\
\hline \multicolumn{5}{|c|}{ Cancer stage at original diagnosis, $n$} \\
\hline $\mathrm{Ta}$ & 12 & 7 & $22^{\mathrm{a}}$ & $4 I^{a}$ \\
\hline TI & 8 & 10 & 2 & 20 \\
\hline Carcinoma in situ & 2 & 2 & $2^{\mathrm{a}}$ & $6^{\mathrm{a}}$ \\
\hline Positive cytology & 0 & 3 & 0 & 3 \\
\hline \multicolumn{5}{|c|}{$\begin{array}{l}\text { Cancer grade at original diagnosis (World Health Organization } 1973 \\
\text { grading system), n }\end{array}$} \\
\hline 1 & 6 & 3 & 9 & 18 \\
\hline 2 & 6 & 4 & 8 & 18 \\
\hline 3 & 10 & 15 & 8 & 33 \\
\hline \multicolumn{5}{|l|}{ Risk group for recurrence, $n$} \\
\hline High & 16 & 15 & 16 & 47 \\
\hline Intermediate & 2 & 4 & 1 & 7 \\
\hline Low & 4 & 3 & 8 & 15 \\
\hline \multicolumn{5}{|l|}{ Previous intravesical treatment, $\mathrm{n}$} \\
\hline Bacillus Calmette-Guérin & 13 & 14 & 10 & 37 \\
\hline Mitomycin C & 5 & 3 & 0 & 8 \\
\hline None & 4 & 5 & 15 & 24 \\
\hline $\begin{array}{l}\text { Mean time since diagnosis of } \\
\text { bladder cancer, years }\end{array}$ & 12 & 4 & 8 & 8 \\
\hline Mean previous cystoscopies, $\mathrm{n}$ & 8.9 & 8.0 & 14.7 & 10.8 \\
\hline $\begin{array}{l}\text { Mean previous transurethral } \\
\text { resections of the bladder, } \mathrm{n}\end{array}$ & 3.4 & 1.7 & 3.2 & 2.8 \\
\hline
\end{tabular}


Table 2 Logistics of blue light flexible cystoscopy

\begin{tabular}{|c|c|c|c|c|}
\hline Parameter & Uppsala & Malmö $^{a}$ & Bærum & Total $^{\mathrm{a}}$ \\
\hline \multicolumn{5}{|c|}{$\begin{array}{l}\text { Time required per patient for preparation and instillation of } \\
\text { hexaminolevulinate, } n\end{array}$} \\
\hline$\leq 10$ minutes & 16 & 22 & 19 & 57 \\
\hline$>10-\leq 15$ minutes & 6 & 0 & 5 & 11 \\
\hline$>15-\leq 20$ minutes & 0 & 0 & I & 1 \\
\hline $\begin{array}{l}\text { Total waiting time per patient } \\
\text { between HAL instillation and } \\
\text { cystoscopy, minutes }\end{array}$ & 59 & 55 & 60 & 59 \\
\hline \multicolumn{5}{|l|}{ Cystoscopy time, $\mathrm{n}$} \\
\hline$\leq 5$ minutes & I & 6 & 6 & 13 \\
\hline$>5-\leq 10$ minutes & 14 & 14 & 8 & 36 \\
\hline$>10-\leq 15$ minutes & 8 & 2 & 11 & 21 \\
\hline Patients per hour & 2 & 2 & 2 & 2 \\
\hline
\end{tabular}

Note: ancludes one patient who had blue light flexible cystoscopy twice. Abbreviation: HAL, hexaminolevulinate.

had an additional waiting time of 45-60 minutes between instillation and examination, compared with the time required for standard follow-up flexible cystoscopy with white light only. Patients did not require physician or nurse supervision during this time. Patients were discharged immediately after BLFC. Improvements in efficiency over time, such as reduced time required for preparation and cystoscopic examination, were observed for physicians and nurses initially new to the equipment, as they became more familiar with the equipment and the procedure. With two cystoscopes available and adequate planning, it was possible to perform BLFC on two patients per hour in this outpatient setting.

The BLFC equipment provided high-quality images. Across the three centers, histologically confirmed bladder tumors were identified in 11 patients with both white light flexible cystoscopy and BLFC, whereas three patients had lesions identified only by BLFC (Table 3). The lesions identified by BLFC only were: two Ta tumors in two patients (both $5 \mathrm{~mm}$ diameter) and CIS in one patient, who had prior history of CIS. More and smaller lesions were detected with BLFC than with white light flexible cystoscopy, and tumor margins were more clearly visible under blue light (Figure 1). The benefit of BLFC was consistently seen across all study centers.

In total, 69 biopsies were taken from 45 patients. With the equipment used for BLFC, it was usually possible to take good-quality biopsies, allowing adequate pathological analysis of the tissue. A known limitation of flexible cystoscopy is the inability to reach some lesions for biopsy because of insufficient bending of the scope; this issue was experienced in one case in this study, where the suspected lesion was situated ventrally. Eleven of the patients in whom lesions were detected could be managed with fulguration, while

Table 3 Detection and management of recurrent tumors in the current study

\begin{tabular}{|c|c|c|c|c|c|}
\hline \multicolumn{2}{|l|}{ Parameter } & Uppsala & Malmö & Bærum & Total \\
\hline \multicolumn{6}{|c|}{ Patients with malignant lesions identified in this study, $n$} \\
\hline \multirow[t]{2}{*}{ Any stage } & BLFC & 5 & 6 & 3 & 14 \\
\hline & WLFC & 4 & 5 & 2 & 11 \\
\hline \multirow[t]{2}{*}{ Ta lesions } & BLFC & 3 & 2 & 2 & 7 \\
\hline & WLFC & 2 & 2 & I & 5 \\
\hline \multirow[t]{2}{*}{ TI lesions } & BLFC & 1 & 2 & 0 & 3 \\
\hline & WLFC & I & 2 & 0 & 3 \\
\hline \multirow[t]{2}{*}{ Carcinoma in situ } & BLFC & I & 2 & I & 4 \\
\hline & WLFC & 1 & I & I & 3 \\
\hline \multicolumn{6}{|c|}{ Management of detected lesions, $n$} \\
\hline \multicolumn{2}{|l|}{ Fulguration } & 5 & 4 & 2 & 11 \\
\hline \multicolumn{2}{|c|}{ Referral for TURB } & 0 & 2 & $\mathrm{I}$ & 3 \\
\hline
\end{tabular}

Abbreviations: BLFC, blue light flexible cystoscopy; TURB, transurethral resection of the bladder; WLFC, white light flexible cystoscopy.
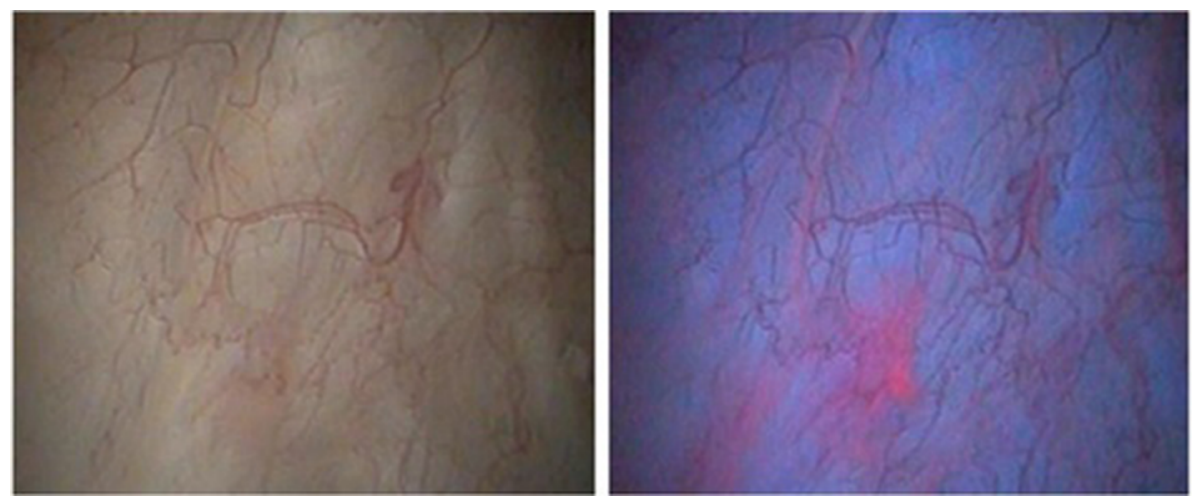

Figure I Cystoscopic appearance of bladder tumors under white light (left) and blue light (right). Images are from a 75-year-old man with a 4-year history of bladder cancer (most serious diagnosis Ta grade I), who was found to have carcinoma in situ on follow-up flexible cystoscopy that was clearly visible under blue light. Image credit: Reza Zare, MD, Urologist, Head of Urology Department, Bærum Hospital Vestre Viken HF, Oslo, Norway. 
only three required referral to the operating room for TURB. TURB referral was due to multiple (seven) recurrences in one patient; indication of advanced tumor in one patient; and suspected papilloma $>5 \mathrm{~mm}$ plus multiple ( $>3$ ) tumors $<5$ $\mathrm{mm}$ in diameter and suspected CIS in one patient.

No patients reported tolerability issues with the BLFC procedure, and no adverse events attributable to BLFC or hexaminolevulinate instillation were reported. Although cystoscopy, biopsy, and fulguration were performed without general anesthesia, few patients reported discomfort during the procedure. One patient became ill after the biopsy, considered to be a stress reaction. In this case, the procedure was terminated, and the patient recovered rapidly.

\section{Discussion}

Across the three centers participating in this study, overall findings were consistent in reporting that routine use of BLFC was feasible in the outpatient setting. BLFC improved the detection of bladder cancer recurrence compared with white light flexible cystoscopy, with recurrent lesions identified in 14 and 11 patients, respectively; thus, three patients were mistakenly defined as tumor free using white light flexible cystoscopy. It may be debated whether further lesions could be detected if the bladder was viewed for a second time under white light - ie, whether the detection benefit comes from the extra time spent on the second review of the bladder, regardless of whether blue light or white light is used. However, the benefit of hexaminolevulinate-guided blue light cystoscopy over white light cystoscopy has been well established, ${ }^{8,9}$ including in a randomized Phase III clinical trial. ${ }^{8}$ This benefit is also seen in the setting of good-quality white light cystoscopy, ${ }^{19}$ indicating that the benefit derives from the technology itself and not the time spent viewing the bladder.

Importantly, the equipment used in this study allowed good-quality biopsies to be taken, providing sufficient tissue for pathologic analysis. It was also feasible to identify small lesions with BLFC, which could then be managed by fulguration in the outpatient setting. BLFC could be of value in identifying early recurring lesions that are not always visible under white light, while they can still be managed with fulguration. At the same time, early identification of non-focal CIS or large lesions that could not be managed in the outpatient department allowed patients to be referred promptly for appropriate inpatient management. The BLFC procedure was well tolerated, with no adverse events reported during the study. Some patients experienced minor discomfort during biopsy and fulguration, which, however, did not affect their willingness to undergo the procedure. Several patients spontaneously expressed greater confidence in their tumor-free status when assessed using BLFC compared with white light flexible cystoscopy.

There is a concern that BLFC may not lend itself readily to the outpatient setting because of the time required for photosensitizer instillation, limitations in biopsy ability, and need for rapid cleaning of the equipment. We found that the limiting factors were the number of cystoscopes available and facilities for cleaning equipment. With good planning, the availability of two blue light cystoscopes and standard equipment cleaning facilities allowed two patients per hour to be managed using BLFC. Patients did not need to be supervised between instillation of hexaminolevulinate and cystoscopy, and they were free to leave the department until the cystoscopy was scheduled. Staff was therefore able to carry out other activities in the outpatient department after the instillation process. The BLFC equipment is generally similar to the equipment for white light flexible cystoscopy, and minimal specific training was required for urologists and nurses at the three centers, other than tips on using the equipment and the cystoscopy procedure. There was somewhat of a learning curve for the staff, typically over the first four or five patients, and the value and importance of familiarity with the equipment and procedure were demonstrated by the efficiency gains made over the course of the study. For example, presence of urine during BLFC results in green fluorescence, which obscures visibility. The flexible cystoscope used in the study was equipped with a suction port, allowing the examiner to rinse the bladder during cystoscopy and maintain a clear field of view. Identifying the best method of rinsing the bladder formed a part of the learning curve for the procedure, with BLFC performed more efficiently as the urologists gained experience and improved their technique. Given these efficiency gains, examination of consecutive patients in dedicated BLFC clinic sessions should help to ensure that staff receives sufficient experience with the cystoscopy equipment and procedures to maximize efficiency and maintain optimal use of resources.

The maximum number of biopsies that can be taken is around five, as bleeding then blurs the vision. Potential difficulties in accessing lesions for biopsy can be addressed using different thickness of biopsy forceps, which can overcome issues with bending of the scope in the biopsy procedure.

Although the current study is limited by its nonrandomized design and small sample size, the findings are consistent with the published literature,,$^{7,13-17}$ and support the US and European expert consensus views ${ }^{9,11}$ that BLFC could have a role in monitoring of bladder cancer recurrence in the 
outpatient setting. As most recurrences of bladder cancer are reported between 3 and 24 months after initial TURB, BLFC could be used in the early follow-up period to ensure prompt diagnosis of recurrent disease and minimize longterm sequelae of insufficiently managed disease. Various subgroups of patients with bladder cancer could benefit from the use of BLFC during follow-up. For low-risk patients, the long-term burden of disease could be reduced through early detection and outpatient management of any recurrences, eg, using improved laser technology, ${ }^{3}$ or through increased intervals between follow-up cystoscopies as a result of confidence in the disease-free status. For intermediate- and high-risk patients, early identification and appropriate management in the outpatient setting or rapid referral to the operating room allow recurrent disease or CIS to be managed effectively, thereby reducing the risk of further recurrence or progression. In elderly patients and those in whom surgery with general anesthesia is unsuitable, BLFC could help to avoid any complications associated with management of the disease in the operating room, without compromising detection of recurrence or follow-up care.

A health economic model has recently been developed to explore the cost consequences of using hexaminolevulinateguided BLFC in the outpatient follow-up of patients with NMIBC. The model, based on the Swedish health care system, indicates that BLFC would allow more outpatient treatment with improved detection of recurrent tumors, resulting in reduced need for TURB, cystectomies, bed days, and operating room time. ${ }^{20}$ BLFC use across all risk groups would have minimal budget impact $(+1.6 \%$ total cost $)$ over 5 years and would achieve cost savings in intermediate- and high-risk groups from year 2. ${ }^{20}$ The actual cost benefit of BLFC in different health care settings needs to be confirmed in real-world studies.

\section{Conclusion}

Routine use of BLFC in the outpatient setting is feasible. It seems to improve the detection of bladder cancer recurrence compared with white light flexible cystoscopy. As a result, small tumors can be managed immediately with fulguration, avoiding the need for referral to the operating room for rigid cystoscopy and TURB under general anesthesia. Logistically, little training appears to be required for urologists and nurses, and, once health care professionals become familiar with the procedure, patient flow is smooth. Therefore, BLFC in the outpatient setting offers potential clinical, economic, and patient benefits in the follow-up of patients with NMIBC. A prospective randomized trial is necessary to confirm the impact of this new approach compared with current standards.

\section{Acknowledgments}

The authors wish to thank the staff who provided support during procedures at the outpatient clinics: Jeanette Bylund in Uppsala and Eva Broström in Malmö, and Dr Tina Kornmo, Dr Christian Arstad, Anne Storheil, Marit Berg Nyquist, and Vigdis Johnsen in Bærum.

Writing assistance in the preparation of this paper was provided by Parexel and Succinct Medical Communications, which was funded by Photocure ASA, Oslo, Norway.

\section{Author contributions}

Reza Zare, Magnus Grabe, and Per-Uno Malmström had full access to the data for their respective centers and take full responsibility for the integrity of the data. Gregers $G$ Hermann acted as a technical adviser during the study. All authors contributed toward data analysis, drafting and critically revising the paper, gave final approval of the version to be published, and agree to be accountable for all aspects of the work.

\section{Disclosure}

All authors have provided services as speakers in meetings funded by Photocure ASA, Oslo, Norway, and have acted as advisers for Photocure. The authors report no other conflicts of interest in this work.

\section{References}

1. Babjuk M, Böhle A, Burger M, et al. Guidelines on Non-Muscle-Invasive Bladder Cancer (Ta, T1 and CIS). Arnhem, The Netherlands: European Association of Urology; 2016.

2. Liedberg F, Hagberg O, Holmäng S, et al. Local recurrence and progression of non-muscle-invasive bladder cancer in Sweden: a populationbased follow-up study. Scand J Urol. 2015;49(4):290-295.

3. Hermann GG, Mogensen K, Lindvold LR, Haak CS, Haedersdal M. Office-based transurethral devascularisation of low grade non-invasive urothelial cancer using diode laser. A feasibility study. Lasers Surg Med. 2015;47(8):620-625.

4. Hermann GG, Mogensen K, Toft BG, Glenthøj A, Pedersen HM. Outpatient diagnostic of bladder tumours in flexible cystoscopes: evaluation of fluorescence-guided flexible cystoscopy and bladder biopsies. Scand J Urol Nephrol. 2012;46(1):31-36.

5. Yeung C, Dinh T, Lee J. The health economics of bladder cancer: an updated review of the published literature. Pharmacoeconomics. 2014;32(11):1093-1104.

6. Yoshimura K, Utsunomiya N, Ichioka K, Matsui Y, Terai A, Arai Y. Impact of superficial bladder cancer and transurethral resection on general health-related quality of life: an SF-36 survey. Urology. 2005;65(2):290-294.

7. Mogensen K, Christensen KB, Vrang ML, Hermann GG. Hospitalization for transurethral bladder resection reduces quality of life in Danish patients with non-muscle-invasive bladder tumour. Scand J Urol. 2016;50(3):170-174. 
8. Daneshmand S, Patel S, Lotan Y, et al; Flexible Blue Light Study Group Collaborators. Efficacy and safety of blue light flexible cystoscopy with hexaminolevulinate in the surveillance of bladder cancer: a phase III, comparative, multicenter study. J Urol. 2018;199(5): 1158-1165.

9. Burger M, Grossman HB, Droller M, et al. Photodynamic diagnosis of non-muscle-invasive bladder cancer with hexaminolevulinate cystoscopy: a meta-analysis of detection and recurrence based on raw data. Eur Urol. 2013;64(5):846-854.

10. Witjes JA, Babjuk M, Gontero P, et al. Clinical and cost effectiveness of hexaminolevulinate-guided blue-light cystoscopy: evidence review and updated expert recommendations. Eur Urol. 2014;66(5): 863-871.

11. Daneshmand S, Schuckman AK, Bochner BH, et al. Hexaminolevulinate blue-light cystoscopy in non-muscle-invasive bladder cancer: review of the clinical evidence and consensus statement on appropriate use in the USA. Nat Rev Urol. 2014;11(10):589-596.

12. Malmström PU, Grabe M, Haug ES, et al. Role of hexaminolevulinateguided fluorescence cystoscopy in bladder cancer: critical analysis of the latest data and European guidance. Scand J Urol Nephrol. 2012;46(2):108-116.

13. Witjes JA, Moonen PM, van der Heijden AG. Comparison of hexaminolevulinate based flexible and rigid fluorescence cystoscopy with rigid white light cystoscopy in bladder cancer: results of a prospective phase II study. Eur Urol. 2005;47(3):319-322.
14. Loidl W, Schmidbauer J, Susani M, Marberger M. Flexible cystoscopy assisted by hexaminolevulinate induced fluorescence: a new approach for bladder cancer detection and surveillance? Eur Urol. 2005;47(3):323-326.

15. Wong KA, Zisengwe G, Athanasiou T, O’Brien T, Thomas K. Outpatient laser ablation of non-muscle-invasive bladder cancer: is it safe, tolerable and cost-effective? BJU Int. 2013;112(5):561-567.

16. Bertrand J, Soustelle L, Grès $\mathrm{P}$, et al. Intérêt de la vidéocystoscopie souple en lumière bleue $\left(+\mathrm{Hexvix}^{\circledR}\right)$ en consultation pour le diagnostic des tumeurs de vessie [Interest of flexible videocystoscopy in blue light $\left(+\right.$ Hexvix $\left.^{\circledR}\right)$ in consultation for the diagnosis of vesical tumor]. Prog Urol. 2012;22(3):172-177. French.

17. Karl A, Weidlich P, Buchner A, et al. Photodynamic diagnosis of the urinary bladder using flexible instruments - ready for the outpatient setting? J Clin Trials. 2014;4:190.

18. Lindvold LR, Hermann GG. An optical method for reducing green fluorescence from urine during fluorescence-guided cystoscopy. Methods Appl Fluoresc. 2016;4(4):045002.

19. Mariappan P, Rai B, El-Mokadem I, et al. Real-life experience: early recurrence with Hexvix photodynamic diagnosis-assisted transurethral resection of bladder tumour vs good-quality white light TURBT in new non-muscle-invasive bladder cancer. Urology. 2015;86(2):327-331.

20. Dansk V, Malmström PU, Bläckberg M, Malmenäs M. Hexaminolevulinate hydrochloride blue-light flexible cystoscopy in the detection and follow-up of nonmuscle-invasive bladder cancer: cost consequences during outpatient surveillance in Sweden. Future Oncol. 2016;12(8):1025-1038.
Research and Reports in Urology

\section{Publish your work in this journal}

Research and Reports in Urology is an international, peer-reviewed, open access journal publishing original research, reports, editorials, reviews and commentaries on all aspects of adult and pediatric urology in the clinic and laboratory including the following topics: Pathology, pathophysiology of urological disease; Investigation and treatment of

\section{Dovepress}

urological disease; Pharmacology of drugs used for the treatment of urological disease. The manuscript management system is completely online and includes a very quick and fair peer-review system, which is all easy to use. Visit http://www.dovepress.com/testimonials.php to read real quotes from published authors. 